\title{
GRAVITY FIELD AND OCEAN TIDES MODELING FOR PRECISE ORBIT DETERMINATION OF DORIS SATELLITES
}

\author{
Petr ŠTĚPÁNEK ${ }^{1)}$ *, Aleš BEZDĚK ${ }^{2)}$, Jan KOSTELECKÝ ${ }^{1)}$ and Vratislav FILLER ${ }^{1)}$ \\ ${ }^{1)}$ Research Institute of Geodesy, Topography and Cartography, Ústecká 98, 25088, Zdiby, Czech Republic \\ ${ }^{2)}$ Astronomical Institute, Czech Academy of Sciences, Fričova 298, 25165 Ondřejov, Czech Republic
}

*Corresponding author's e-mail: petr.stepanek@pecny.cz

\begin{tabular}{l} 
ARTICLE INFO \\
\hline Article history: \\
Received 13 April 2015 \\
Accepted 14 September 2015 \\
Available online 5 November 2015 \\
\hline
\end{tabular}

Keywords:

DORIS

POD

Gravity field truncation degree

Ocean tides

Time variable gravity

\begin{abstract}
Gravitational forces are the major sources of perturbing accelerations acting on satellites in low Earth orbits. Recently, the modeling of the global Earth gravity field strongly benefited from the satellite gravity missions CHAMP, GRACE and GOCE. Besides the static gravity models also the time-variable models are now available, including the seasonal and linear (or piecewise linear) terms. We focus on the gravity modeling for precise orbit determination (POD) using DORIS (Doppler Orbitography and Radiopositionning Integrated by Satellite) data. First we show the relative effect of various gravitational and non-gravitational perturbation forces on orbits of Earth artificial satellites at several different altitudes (460-5900 km; satellites Swarm A, SPOT-5, Jason-2, Lageos-1). Then we study the impact of a particular setting (maximum degree, time-variable terms) of gravity field and ocean tide models on the quality of the determined orbits. For DORIS satellites SPOT-5 and Jason-2, we optimized the geopotentical coefficient truncation degree to meet the limit of $1 \mathrm{~mm}$ radial orbit error and $2 \mathrm{~mm}$ cross-track and along-track orbit error. A minimum limit for the geopotential coefficient truncation degree is 75 for SPOT-5 and 50 for Jason-2, when using the common dynamic orbit settings and daily orbit arc. The minimum limit for the application of the gravity changes due to the ocean tides is 25 for SPOT-5 and 20 for Jason-2. However, we also demonstrate that these limits depend on an orbit parametrization. Our experiments with SPOT-5 and Cryosat POD show a significant impact of the piecewise linear modeling, applied in the time-varying part of the gravity field model EIGEN-6S2, indicated by the effect on the RMS of the orbit fit. A similar effect of the annual and semiannual gravity terms application on the RMS of the orbit fit was not found, but the arc overlap RMS decreased by $0.6-2 \%$.
\end{abstract}

\section{INTRODUCTION}

Gravitational forces are among the major sources of the perturbing accelerations for the low Earth orbiters (LEO). High accuracy modeling of the gravitational forces is a necessary condition for LEO precise orbit determination (POD). Recently, the modeling of the global Earth gravity field has strongly benefited from the satellite gravity missions CHAMP, GRACE and GOCE (for missions description see e.g. Balmino et al., 1999; Touboul et al., 1999; for gravity field e.g. Bezděk et al., 2014; Austen at al., 2002). The POD using DORIS (Doppler Orbitography and Radiopositionning Integrated by Satellite) data, realized in the framework of the IDS (International DORIS Service) (Willis et al., 2010), reflects these improvements to meet the maximal accuracy. The accuracy of the POD does not affect only the quality of the estimated orbit ephemerides, but also the quantities derived from a free-network solution, mainly the station coordinates and the Earth rotation parameters (ERP). The progress in the global gravity field modeling can be illustrated by the comparison of the recommended POD strategy for the ITRF data reprocessing. For ITRF 2008 (International Terrestrial Reference Frame), the static gravity field was applied (e.g. Le Bail et al., 2010; Otten et al., 2010 and Štěpánek et al., 2010a); for recent ITRF processing
(ITRF 2014), the time-varying gravity modeling is recommended by the IDS, at least for the data since 2002 (Willis et al., in press).

Several comparisons of different gravity field applications for DORIS data processing have been performed. The most recently published study (Rudenko et al., 2014) compared the POD accuracy by making use of different EIGEN gravity field models, with the recommendation to use EIGEN-6S2 for the period 1992-2010. However, all the tested models included the annual and semiannual terms with the same truncation degree and thus the total impact of harmonic modeling was then not the subject of the comparative analyses. Moreover, Rudenko et al. (2014) present a multi-technique POD and do not specially focus on fitting the orbit on DORIS observations (from the used satellites only Envisat and T/P (TOPEX/Poseidon) have installed a DORIS receiver). Other recent DORIS papers compare the POD solutions using different standards, but without special focus on gravity field modeling aspects (e.g. Lemoine and GSC Analysis Team, 2013).

Our study is different from the above mentioned publications. We are not searching an optimum gravity field model, all our results were obtained applying the EIGEN-6S2 model with differing restrictions. We want to understand how precisely we 
Table 1 Selected physical and orbital characteristics of the studied example satellites. The initial epoch gives the starting date of prediction intervals, the results of which are shown in Figures 1-4.

\begin{tabular}{|c|c|c|c|c|c|c|}
\hline Satellite & In orbit since & Initial epoch & Altitude & Inclination & Mass & Dimensions \\
\hline Swarm A & 2013 & $1 / 9 / 2014$ & $460 \mathrm{~km}$ & $87.4^{\circ}$ & $500 \mathrm{~kg}$ & $3.3 \times 1.3 \times 0.8 \mathrm{~m}$ \\
\hline SPOT-5 & 2002 & $1 / 4 / 2012$ & $800 \mathrm{~km}$ & $98.7^{\circ}$ & $3000 \mathrm{~kg}$ & $\begin{array}{l}3 \times 3 \times 5.7 \mathrm{~m} \\
+ \text { solar arrays }\end{array}$ \\
\hline Jason-2 & 2008 & $1 / 4 / 2012$ & $1300 \mathrm{~km}$ & $66^{\circ}$ & $500 \mathrm{~kg}$ & $\begin{array}{l}1 \times 1 \times 4 \mathrm{~m}+\text { solar arrays } \\
1.5 \times 10 \mathrm{~m}\end{array}$ \\
\hline Lageos-1 & 1976 & $1 / 1 / 2012$ & $5900 \mathrm{~km}$ & $109.8^{\circ}$ & $400 \mathrm{~kg}$ & sphere, diameter $60 \mathrm{~cm}$ \\
\hline
\end{tabular}

need to apply a given gravity field model, i.e., what is the limit for the degree of geopotential coefficients to meet an accuracy required. Moreover, we want better understand the effect of the time dependent terms, periodical and piecewise linear. Our interest is the order of their impact on the estimated orbit ephemerides and a possible POD improvement reached by using these terms. In addition, we performed a similar study for the gravity variations due to the ocean tides, applying the FES2004 model (Letellier et al., 2004) with differing restrictions. The main goal of the paper is to provide background information for future considerations of the gravity force modeling for POD, particularly for the corresponding application to DORIS data.

Section 1 (Introduction) is followed by Section 2 (Theoretical background), summarizing the effect of major perturbation forces acting on LEO satellites via calculated orbit predictions. In Section 2.1 the impact of various perturbation forces on a LEO orbit for satellites at different altitudes (Swarm A, SPOT-5, Jason-2, Lageos-1) is given and discussed. Sections 2.2 and 2.3 summarize the theoretical basics of the gravity field and ocean tides modeling. Section 3 is devoted to the description of the applied orbit parametrization. Section 4 includes the tests with the gravity field model. Section 4.1 deals with the minimum limit for the geopotential model degree to be applied in the POD of the DORIS satellites. Section 4.2 considers the time-varying gravity terms, their impact on the satellite orbit and on the POD accuracy. Section 5 provides an application study of ocean tides models. The paper completes with Section 6 , Summary and conclusions.

\section{THEORETICAL BACKGROUND}

\subsection{PERTURBING FORCES}

The objective of this paper is to find an optimum parameter setting for the newly available models of the global gravity field and for the ocean tides models when they are applied in the POD of selected DORIS satellites. In this section we want to show what is the effect of these two perturbing forces in the context of other major gravitational and non-gravitational perturbations. The accuracy of predicting the motion of a satellite depends on what physical forces are taken into account for orbit computation. By means of orbit prediction we demonstrate on examples of several satellites at different altitudes the influence of individual perturbing forces, among which those due to the geopotential and ocean tides. The first three examples are LEO satellites, whose orbital altitude is below $2000 \mathrm{~km}$. For these satellites, the atmospheric drag is acting significantly; although the last example satellite flies higher, there is a slight effect on its motion due to the residual hydrogen atmosphere as well. The selected satellite characteristics are given in Table 1. Swarm A is one of the three satellites of ESA's (European Space Agency) Swarm mission to study the geomagnetic field, which were placed in a low polar orbit in November 2013 (Olsen et al, 2015; https://earth.esa.int/swarm/). To selected DORIS satellites, studied in Sections 3-5, in this section we added Swarm A as an example satellite having an altitude below $500 \mathrm{~km}$. The remote-sensing satellite SPOT-5 (Dagras et al., 1995) was launched into a sun-synchronous orbit at an altitude of $800 \mathrm{~km}$ by CNES (https://earth.esa.int/web/guest/missions/3rdparty-missions/current-missions/spot-5). Oceanographic satellite Jason-2 (Lambin et al., 2010) is a joint CNES/NASA mission, its altitude is $1300 \mathrm{~km}$. Both SPOT-5 and Jason-2 are equipped with the DORIS system. The last example is NASA's geodynamic satellite Lageos-1; it is a spherical, high-density satellite placed at an altitude of $5900 \mathrm{~km}$. The POD and modeling of its orbit has been used to compute the long-term changes in the geopotential coefficients of low degrees. Lageos satellites are also essential for the studies on the terrestrial reference frame and Earth rotation.

In Figures 1-4 we show the relative influence of individual forces acting on selected satellites (cf. Vallado, 2006). For each satellite we computed its nominal orbit by means of numerical integration where all the shown forces were taken into account (Bezděk et al., 2009). The curves then show the effect of each perturbing force in terms of positional difference as a function of time; the logarithmic scale of the y-axis helps to gain an order-of-magnitude idea of the impact of each perturbing force. For a more general description of perturbing forces, see e.g. Montenbruck and Gill (2000).

In the first approximation, the motion of a satellite around the Earth is solved as Kepler's problem, the Earth is taken as a mass point and the satellite with a negligible mass is moving around it, following an ideal Keplerian ellipse. In Figure 1 the curve corresponding to this simple two-body motion 
deviates from the real motion by thousands of kilometers after only a two-day prediction. Similar behavior can be seen for all three LEO satellites (Figs. 1-3), the case of the satellite at a higher altitude of $5900 \mathrm{~km}$ (Fig. 4) points to the fact that the model of the "point-mass Earth" is the closer to the real situation, the farther the satellite is from the Earth.

As it is well known from physical geodesy, the gravity field of the Earth cannot be reduced to that of a point mass, the closest mathematical approximation currently in use is the gravity field generated by an oblate ellipsoid (Fukushima, 2013; Sebera et al., 2013). The geopotential, and its corresponding gravity field, has a complex shape, quantitatively the deviation of the real geopotential from the central field is described by a set of spherical harmonic coefficients (Sect. 2.2). Much better approximation to the real satellite motion is to model the Earth as spherical, but flattened at the poles (see e.g. Montenbruck and Gill, 2000 or Vallado, 2006). Mathematically, this corresponds to using the geopotential as a sum of the central term ('two-body') and the 'oblateness term' $\left(\mathrm{C}_{20} \equiv \mathrm{J}_{2}\right.$ term, see Sect. 2.2). Although this model (labeled as 'two-body $+\mathrm{J}_{2}$ ' in Figs. 1-4) is thousands of kilometers away from the nominal orbit after one day, the flattened Earth model is at least two orders of magnitude better then the simplest 'two-body' model.

The next perturbing force is the atmospheric drag, a force caused by the friction of the satellite body against the air molecules. The density of the atmosphere decreases nearly exponentially with increasing height, so the lower the orbit, the larger is the atmospheric drag force, which causes the progressive decrease in the orbit altitude (see e.g. Bezděk and Vokrouhlický, 2004). In Figures 1-4 the gradual decrease in the magnitude of the drag with increasing height is evident. For the satellite at $460 \mathrm{~km}$ (Fig. 1), the drag is the most important perturbing force causing the deviation of $10 \mathrm{~km}$ after 2 days, whereas for the satellite at $5900 \mathrm{~km}$ (Fig. 4), the action of the atmosphere is almost imperceptible, after 4 days the deviation reaches only a few $\mathrm{cm}$. Atmospheric drag together with solar radiation pressure (see below) rank among the so-called nongravitational or surface forces, whose magnitude depends on the physical characteristics of the satellite body (mass, area, surface properties, etc.). This is in contrast to the forces of gravitational origin, which are conservative and thus depend only on the position of the satellite.

The gravity action of the Sun and Moon causes the so-called lunisolar variations in the satellite motion. Compared to the distance to the Sun and Moon, the example satellites fly rather close to the Earth, unlike the previous forces the lunisolar perturbations have about the same magnitude for all the satellites. In Figures 1-4, they produce a deviation of hundreds of meters after one day.
The gravity attraction of the Sun and Moon acts also on the Earth body, which is deformed, and the changed position of solid and ocean masses generate a measurable gravity signal. The perturbing force induced by tidal changes of the solid Earth is larger than that due to the tidal action of the oceans, the solid Earth tides cause a deviation of $40 \mathrm{~m}$ after 4 days for the satellite at $460 \mathrm{~km}$ (Fig. 1). Going higher this deviation is lower, but not too much, even the orbit of the satellite at $5900 \mathrm{~km}$ (Fig. 4) is changed by $10 \mathrm{~m}$ after 4 days.

In Figures 1-4, a similar deviation as that due to the solid Earth tides is caused by the direct solar radiation pressure. For satellites with large solar panels, which sometimes actively follow the direction to the Sun, however, this perturbing force is highly variable. In our case, the satellites equipped with large solar panels are SPOT-5 and Jason-2 (Figs. 2-3). Apart from the direct solar radiation, there are other two radiation perturbative forces usually taken into account for precise orbital computations, the terrestrial infrared radiation and the solar radiation reflected from the Earth. Their magnitude is smaller by a factor of three or more; therefore, we did not include these two forces into Figures 1-4 not to make them too complex. For more details and a magnitude comparison with other perturbative forces, see e.g. Bezděk (2010, Sect. 2.2).

Next perturbing force is that caused by ocean tides. On average, its action is smaller in magnitude compared to the major perturbing forces; for the satellites in Figures 1-4, the ocean tides cause deviations of 3-10 meters after 4 days. Apparently, the perturbations due to ocean tides do not much decrease with increasing altitude for satellites in the studied height range. This could be explained by the fact that at higher altitudes the satellite motion is affected by larger ocean area.

The last effect shown in Figures $1-4$ is due to seasonal variations of the gravity field model. For each satellite we computed the orbit based on the EIGEN-6S2 model (Rudenko et al., 2014) taking into account the annual and semiannual terms on 1 October 2005; in the figures we draw the difference relative to the nominal orbit, in which the mean 2005 gravity field from EIGEN-6S2 (i.e. with annual and semiannual parts averaged out) was used. This choice was motivated by the fact that in October the time-variable gravity has a local extreme (e.g. Bezděk et al., 2014, Fig. 13). For lower two satellites (Figs. 1 and 2), after one day this 'seasonal gravity' effect reaches the position deviation of 1 meter, and of 0.1 meter for the higher satellites (Figs. 3 and 4).

\subsection{GRAVITY FIELD AND ITS TIME VARIATIONS}

As mentioned in the previous section, since the 1970s the geodynamic satellites like Lageos-1 have allowed the monitoring of time changes in the geopotential coefficients of lowest degrees (e.g., Chen 
et al., 2005). In the last decade, three geodesy missions took place with the aim to improve the knowledge of the global Earth gravity field by different observation techniques. Already the first geopotential models derived from precise GPS orbit positioning of the satellite CHAMP (in orbit 20002010) improved the previous satellite-only geopotential models by an order-of-magnitude (Reigber et al., 2003). Since 2002 the GRACE mission - consisting of two satellites arranged for the low-low satellite-to-satellite tracking -has been producing monthly geopotential models by means of which time-variable gravity can be observed. For the first time, a time series of gravity variations due to continental and regional hydrology changes can be detected from space (Tapley et al., 2004; Kostelecký et al., 2013). Currently, the low degree part of the most accurate static gravity field models (up to degree 150, say) is derived from GRACE mission data (e.g., Pail et al., 2010). Finally, the satellite GOCE (in orbit 2009-2013) measured the gravity gradients in space, which were used to improve the medium degrees in the static geopotential models (Floberghagen et al., 2011). Of these three missions the two, CHAMP and GRACE, were focused on the temporal variations of the gravity field. From precise positioning of satellites equipped with a geodetic-quality GPS receiver, the technique known as high-low satellite-to-satellite tracking, the time-variable gravity signal can be obtained, but currently with a lower space resolution compared to models derived from GRACE (Bezděk et al., 2014; Weigelt et al., 2013). In geodesy as well as in other geosciences, GRACE observations of timevariable gravity have been frequently used; for a review see, e.g., Cazenave and Chen (2010) or Chambers and Schröter (2011).

Usually, the gravitational potential of the Earth is represented as a spherical harmonic series

$$
\begin{aligned}
& V(r, \phi, \lambda)=\frac{G M}{r} . \\
& \cdot\left[1+\sum_{l=2}^{\infty} \sum_{m=0}^{l}\left(\frac{a_{e}}{r}\right)^{l} P_{l m}(\sin \phi)\left(C_{l m} \cos m \lambda+S_{l m} \sin m \lambda\right)\right]
\end{aligned}
$$

where $G M$ is the geocentric gravitational constant, $r, \phi, \lambda$ are the radius, geocentric latitude and longitude, $a_{e}$ is the radius of a reference ellipsoid, $P_{l m}(\sin \phi)$ are the fully normalized associated Legendre functions of the first kind of degree $l$ and order $m, C_{l m}, S_{l m}$ are the geopotential harmonic coefficients. To describe the real field of the Earth, the summation over degree $l$ should extend to infinity, geopotential models derived from different types of observational data are limited by a given maximum value $l_{\max }$. Thus, a geopotential model provides the user with a set of parameters $C_{l m}, S_{l m}$ together with the values of other defining parameters
( $G M, a_{e}$, etc.). In this work, we use the model EIGEN$6 \mathrm{~S} 2$, which is a global satellite-only gravity field model up to degree and order 260 based on data from Lageos, GRACE and GOCE satellites (Rudenko et al., 2014). The EIGEN-6S2 geopotential coefficients up to degree 50 are estimated as time variable; for a given epoch, the model provides the terms for the drift as well as for the annual and semiannual variation. Another currently used model is EGM2008, which is a static combined gravity field model (Pavlis et al., 2012).

\subsection{OCEAN TIDES}

Intensive activity of the space geodesy community in developing global models of ocean tides started in the 1990 s in connection with new altimetric missions, especially with TOPEX/Poseidon. This stems from the fact that the tidal signal is the largest contributor to sea surface height variability and accounts for more than $80 \%$ of the signal variance (Shum et al., 1997). Improved modeling of ocean tides is also important for recent gravity missions CHAMP, GRACE and GOCE, because the ocean tides induce variations in the Earth's geoid by amounts that far exceed the measurement sensitivities, and tidal models must be used to correct for this (Ray et al., 2003). The gravitational effects of ocean tides are usually expressed as periodic variations in the normalized geopotential coefficients

$\left[\Delta C_{l m}-i \Delta S_{i m}\right]=\sum_{f} \sum_{+}^{-}\left(C_{f . l m}^{ \pm} \mp S_{f, l m}^{ \pm}\right) e^{ \pm i \vartheta_{f}(t)}$

where $\left(C_{f . l m}^{ \pm}\right.$and $\left.S_{f, l m}^{ \pm}\right)$are the geopotential harmonic amplitudes for the tide constituent $f$ and $\vartheta_{f}(t)$ is the argument of the tide constituent $f$ (Petit and Luzum, 2010). The double sum on the right-hand side is advantageous, because it separates the component independent of time (geopotential harmonic amplitudes) and the component dependent on time (argument of the tide constituent) which does not depend on the harmonic indices $l, m$. The argument of the tide constituent $\vartheta_{f}(t)$ can be expressed as a linear combination with integer coefficients of the Doodson variables. The Doodson variables are defined through the lunar and solar ephemeris, there are six of them corresponding to periods of a lunar day (1.0035 days), nodical month (27.32 days), tropical year (365.2422 days), lunar perigee period (8.847 years), lunar node period (18.61 years) and solar perigee period (20940 years). In practice, ocean tide models provide parameters that allow the user to compute the amplitudes $\left(C_{f . l m}^{ \pm}, S_{f, l m}^{ \pm}\right)$and for each tide constituent $f$ six integer multipliers, with which one can compute the argument $\vartheta_{f}(t)$. In this work, we used the ocean tide model FES2004 (Letellier et al., 2004). 
Table 2 The orbit parametrization and additional parameters estimated in POD.

\begin{tabular}{llll}
\hline Type of orbit & $\begin{array}{l}\text { Dynamic } \\
\text { (standard) }\end{array}$ & $\begin{array}{l}\text { Dynamic } \\
\text { (drag extended) }\end{array}$ & Reduced dynamic \\
\hline Arc length & 1 day & 1 day & 1 day \\
Constant empirical parameters & N/A & N/A & 2 \\
Harmonic empirical parameters & 4 & 4 & 4 \\
Atmospheric drag & $12\left(6^{*}\right)$ & $48\left(6^{*}\right)$ & 96 along track \\
Solar radiation pressure & 1 & 1 & 0 (constant emp.) \\
Earth direct/indirect rad. pressure a priori & a priori & No \\
Zenith total delay & wet part per path & wet part per path & wet part per path \\
Beacon frequency offset & per satellite path & per satellite path & per satellite path \\
\hline
\end{tabular}

* for satellites of altitude over $1000 \mathrm{~km}$ (Jason-2) only 6 drag parameters per day

\section{ORBIT PARAMETRIZATION}

For the following experiments, it is necessary to describe the used POD standards, mainly the orbit parametrization. All the orbits were estimated solely from DORIS observations. The DORIS data were processed by the DORIS development version of the Bernese GPS software (Dach et al., 2007), used at the Geodetic Observatory Pecny (GOP) DORIS analysis center (Štěpánek et al., 2010b). The estimated orbit arc length was 24 hours. In following experiments the data were used from Jason-2 (altitude $1330 \mathrm{~km}$ ), Hy2A $(970 \mathrm{~km})$, SPOT-5 $(830 \mathrm{~km})$ and Cryosat-2 $(730 \mathrm{~km})$. The parametrization of the orbit is included in Table 2. Three different parametrizations are included: standard dynamic parametrization, dynamic parametrization with an increased number of drag parameters and reduced-dynamic orbit parametrization. For most experiments described below, the standard dynamical parametrization was used. A similar approach is the most common in POD performed by the IDS analysis groups (http://idsdoris.org/contribution-itrf2013.html). According to the study presented by Štěpánek et al. (2014), the dynamic parametrization with an extended number of drag parameters is better suited for the free network solution and to improve the accuracy of the estimated parameters, but estimating the drag scaling parameters more frequently than once per revolution may lead to the loss of the physical meaning (i.e., the drag parameters might absorb mismodeling errors of some other perturbation forces). The reduced-dynamic approach was used by GOP IDS analysis center until 2013, when a proper LEO dynamical orbit modeling was not available in the data processing software. The missing exact models of the non-conservative forces were compensated by setting a higher number of empirical and stochastic parameters.

\section{GRAVITY FIELD}

\subsection{LIMIT FOR THE DEGREE OF THE MODEL GEOPOTENTIAL COEFFICIENTS}

In POD, the gravity field models are used with the truncation degree. In DORIS data processing, analysis groups apply different truncation degree limits, the most common value for the maximum degree being 100-120 (http://ids-doris.org/contributionitrf2013.html), the highest limit of 200 is used with the IGN analysis center solutions (Willis and Kuzin, 2014). However, to our knowledge only early studies of dependence of the orbit error on the gravity filed application are available (Nerem et al., 1990; Rapp and Pavlis, 1990). Accuracy of the orbit determination dramatically increased during last 25 years and a new study is needed. Our interest is to determine a reasonable limit to avoid underestimation, which would affect the solution accuracy, or overestimation, leading to a waste of the data processing computer time. First of all, we processed one year of data (2012) several times with different gravity field model limitations, set by a different limit for the geopotential coefficient truncation degree. Figure 5 presents the calculated RMS values of the orbit differences estimated applying the different gravity field truncation degree, where an orbit estimated with the gravity field model up to degree 150 is used as a reference. The RMS values are plotted for satellites SPOT-5 and Jason-2, i.e., one "low" and one "high" DORIS satellite. A possible way, how we can state the requested accuracy limit of the gravity field application, is to define a maximum acceptable Mean and RMS of the estimated orbit differences. The reasonable values of the mean difference could be two orders of magnitude lower than the precision of the POD, the reasonable values for orbit difference RMS (after mean difference removal) could be one order of magnitude lower than the precision of the POD. We define the acceptable limit for the mean difference as $0.1 \mathrm{~mm}$ in the radial and $0.2 \mathrm{~mm}$ in the along-track and cross-track directions and limit for RMS of the orbit differences as $1 \mathrm{~mm}$ in the radial and $2 \mathrm{~mm}$ in the along-track and cross-track directions. According to our results such a RMS request is fulfilled by applying the gravity field model up to degree 75 for SPOT-5 and up to degree 50 for Jason-2 at least for 1-day orbit arc. Clearly, the estimated orbit of SPOT-5 is more affected by the choice of the maximum degree due to the lower satellite altitude. The mean orbit differences relative to the reference (not shown here) are not so sensitive to the degree limit and for the SPOT-5 orbit estimated with maximum geopotential 


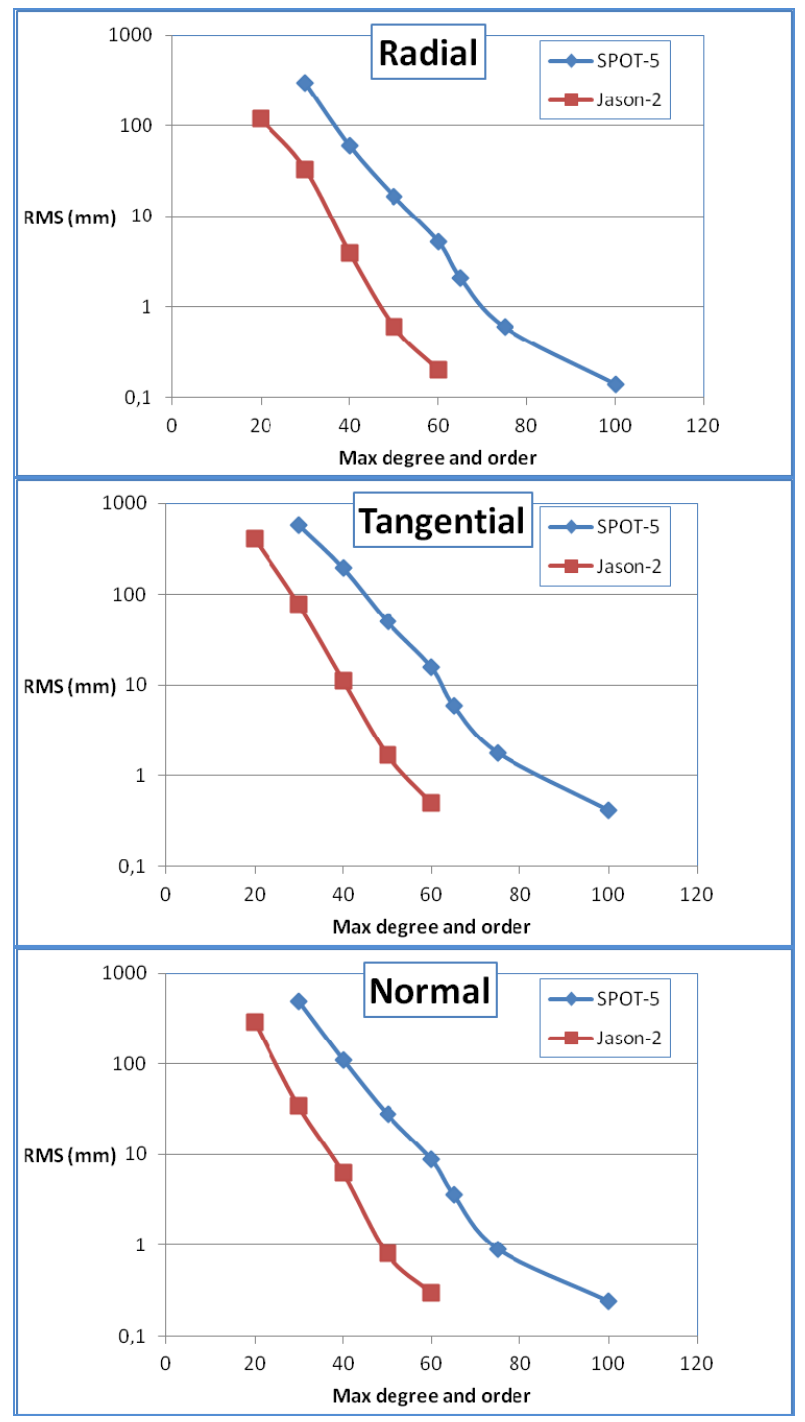

Fig. 5 RMS of the SPOT-5 and Jason-2 orbits differences, the orbits are estimated with various limits on the geopotential model maximum degree. The orbit with the gravity field model applied up to degree 150 is used as the reference.

degree 75 reach less than $0.1 \mathrm{~mm}$ in the radial and normal directions and around $0.2 \mathrm{~mm}$ in the tangential direction. For the Jason-2 orbit estimated with maximum geopotential degree 50 , the mean difference reaches less than $0.1 \mathrm{~mm}$ in the radial and normal directions and $-0.2 \mathrm{~mm}$ in the tangential direction.

These orbit comparison statistics relate to a particular orbit parametrization, and as such may not be relevant for orbits approximated by a different number of parameters, or with parameters defined in a different way. In the above presented testing, we used the "standard dynamic" orbit parametrization of the daily orbit arc, described in Table 2, consisting of totally 21 orbit parameters (12 drag parameters) for SPOT-5 and 17 parameters (6 drag parameters) for Jason-2. Twelve drag parameters per day represent the most common drag parametrization in the framework

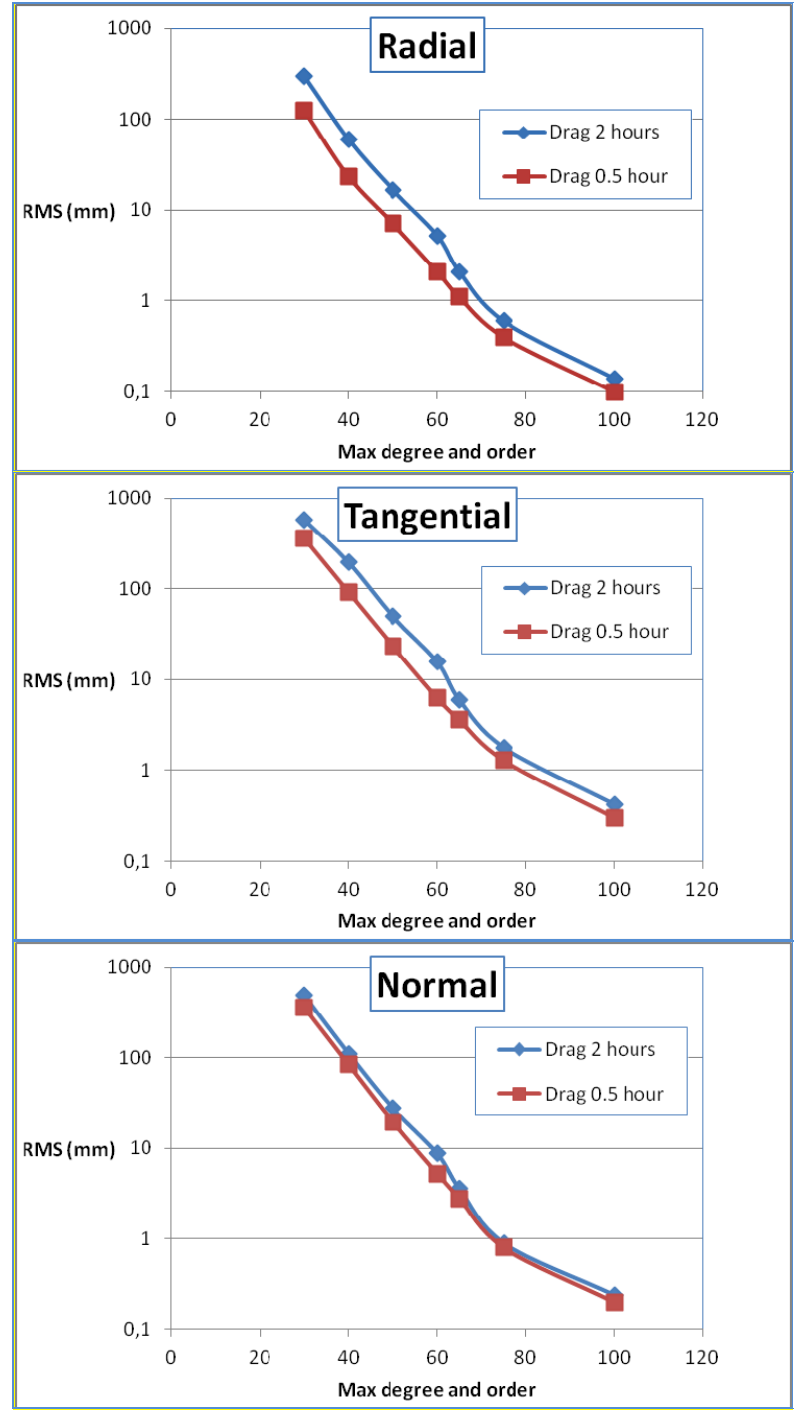

Fig. 6 RMS of the SPOT-5 orbit difference, orbit estimated with various limits on the geopotential model maximum degree and with different number of the adjusted drag parameters. The orbit with the gravity field model applied up to degree 150 is used as the reference.

of the current IDS analysis centers processing (http://ids-doris.org/contribution-itrf2013.html) for DORIS satellites with an altitude of around $800 \mathrm{~km}$ (e.g. SPOT-5). Gobindass et al. (2010) recommend to estimate a drag scaling coefficient for these satellites at least each 2 hours, compensating the uncertainties in the atmosphere density modeling. At the Jason-2 altitude, the atmosphere drag effect is much lower and such frequent handling of the drag scaling parameters is not needed. For SPOT-5 we repeated the tests also with 48 drag parameters, recommended by Štěpánek et al. (2014), which increased the total number of the orbit parameters to 59. This parametrization is included in Table 2 as "drag extended". The comparison of the orbit differences RMS for both approaches is shown in Figure 6. For the orbit estimation with the gravity field coefficients up to 
degree 75 , the ratio of the RMS of the orbit differences with 12 drag parameters and of that with 48 drag parameters is 1.50 in the radial, 1.36 in the tangential and 1.12 in the normal directions. The correlation between the number of the orbit parameters and the orbit difference RMS is expected, since a part of the gravity field mismodeling could be absorbed by the additional parameters. The very frequently estimated drag scaling parameters then, however, lose their physical meaning.

When performing POD, the upper limit for the geopotential coefficient degree may significantly affect the machine computing time. For the last ITRF DORIS data re-processing, each analysis center has to re-process all the DORIS data since $1^{\text {st }}$ January of 1992 up to $31^{\text {st }}$ August 2014. For different reasons, some periods of data have been reprocessed several times during the evolution of DORIS combined solutions. The necessary processing time strongly depends on the used hardware and software and can differ from center to center. At GOP analysis centre, for example, processing one year of data for two satellites takes more than 24 hours of the machine time when using the four $2.3 \mathrm{GHz}$ processor dual core machine. Figure 7 displays the percentage of the saved processing time as a function of the maximum geopotential coefficient degree relative to the solution with the degree limit of 150 . The limits 75 for SPOT5 and 50 for Jason-2, recommended above, save about $25 \%$ of the computing time. When we consider solutions with limit 120 as the reference, then the limits 75 for SPOT-5 and 50 for Jason-2 save both around $11 \%$ of the machine time.

\subsection{TIME VARIABLE GRAVITY MODELING}

Application of the drifts for the pre-GRACE period, where the gravity field time varying model is based on the Lageos data and other less accurate data, could be a source of the modeling errors (here we mean the drifts for the lower part up to degree 50 of the model EIGEN-6S2; the long-term behavior of degree two coefficients determined from Lageos-1/2 SLR tracking data are rather reliable, see e.g. Cheng et al., 2011). Since the 2000's also the drifts of the geopotential coefficients have been estimated and added to the selected model coefficients, initially only for $\mathrm{C}_{20}$ and some others. The gravity field model EIGEN-6S2, nowadays the gravity field model most frequently used by IDS, contains the piecewise linear approximation of the time variable mean signal for coefficients up to degree 50 , besides the annual and semiannual periodic terms. In other words, the mean signal is modeled by the bias and the drift for each year of the relevant time period, with continuity constraints. The application of the time variable gravity terms is a part of the current DORIS data IDS processing standards. The improvement of the DORIS solutions applying the recent standards is documented (e.g. Lemoine et al., 2014), but the time varying gravity field impact is not analyzed separately from

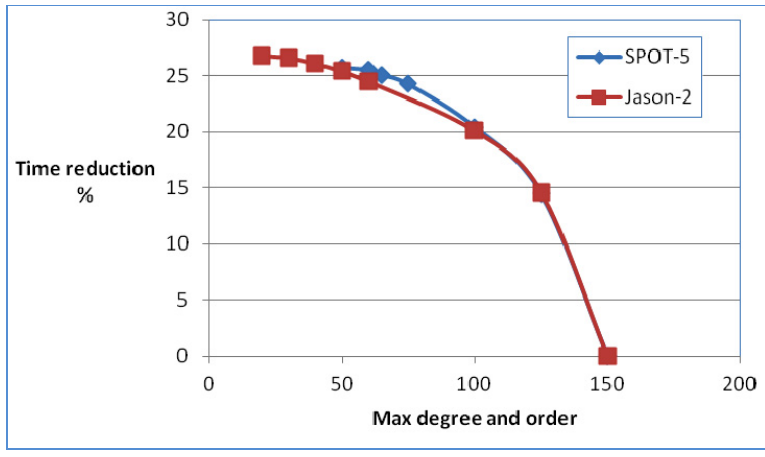

Fig. 7 Reduction in the machine computing time.

other solution improvements. Couhert et al. (2015) compares the Jason $(-1,-2)$ radial orbit component determined applying the time varying gravity field approximated by drift and seasonal signal on one side and the 10-days gravity field series on the other side. The paper focuses on the estimation of the regional distribution of the seasonal orbit error due to the gravity modeling inaccuracy, employing the independent orbit SLR residuals. Another recent study (Melachroinos et al. 2014) focuses on the sensitivity of GPS satellite orbits (much higher altitude than for DORIS satellites) on time varying gravity field.

Rudenko et al. (2014) shows on long data series that the application of the coefficient drifts has a minor impact on the RMS of the DORIS orbit fit, but for DORIS the RMS decrement is less significant than that of SLR. Rudenko et al. (2014) used DORIS data only from altimetry satellites TOPEX/Poseidon (T/P) and Envisat, moreover most of the $\mathrm{T} / \mathrm{P}$ processed data came from the pre-GRACE period. In the present paper, we analyze pure DORIS solutions and not the multi-technique POD. Besides the drifts we separately analyze also the influence of annual and semiannual terms. Moreover, we use different satellites and we are interested not only in the impact on the accuracy, but also in the size of the orbit differences due to the application of the time variable gravity.

\subsubsection{PIECEWISE LINEAR MODELING}

In the gravity field model EIGEN-6S2, each coefficient up to degree 50 is represented by its constant value (bias) and drift for each year and by the corresponding amplitudes of the annual and semiannual terms. However, such a coefficient parametrization is the most representative only for the period covered by GRACE data used for the model estimation, in this case 2002.0-2012.0. For the POD and free-network solutions, we often need to process very recent data out of the gravity field modeling interval or older data from the pre-GRACE period (e.g. free-network solutions for ITRF DORIS data reprocessing have been performed from all cumulative DORIS data since 1992). Application of the drifts for the pre-GRACE period could be a source of the 
modeling errors. Thus, outside the above mentioned interval we can only use the static gravity field or to apply inaccurately determined drifts or their extrapolation.

The static models are usually accompanied by their respective reference epoch. Expected differences between the application of the static and piecewise linear gravity field then depend on the time interval between the static model reference epoch and the time of comparison. Similarly, the effect of neglecting the gravity field variations outside of the relevant piecewise linear modeling time span (typically GRACE period) depends on the time interval between the epoch of interest and the time boundary of the variable gravity modeling period. There are several ways how to test this dependence. We can compute the POD estimates for both the static and the timevarying model and compare the estimated orbits and the corresponding statistics. The advantage of this approach is the direct comparison between the application of the two gravity field models. Another possibility, which we follow in this study, is to use only the time-varying field, but to reprocess the orbits with the values of the gravity field coefficients interpolated for some other time point, different from the epoch of the estimated orbits. The advantage of this approach is that the satellite orbits can be evaluated repeatedly with different gravity field reference points, while the same set of observations is used for the orbit fits. Then we have solutions with different gravity field reference points, but evaluated with the same observations. The statistics obtained for different gravity field reference points can be directly compared.

We processed 3 months of the SPOT-5 DORIS data (May-July 2011) with different gravity field model settings. First, we applied the model EIGEN6S2 including all the terms, limited only by the coefficient degree truncation degree (100). Second, we processed the same data again, but with the geopotential coefficients interpolated for the epoch subsequently 1, 2, 3, 4 and 5 years before the observation epoch. Figure 8 displays the mean and RMS of the orbit differences estimated with shifting piecewise linear changes by 1-5 years compared to the nominal orbit estimated with a standard application of the gravity field model. The radial and normal mean differences reach values up to $0.5 \mathrm{~mm}$. The tangential mean difference is larger and by neglecting the drifts for 5 years it reaches more than $5 \mathrm{~mm}$. Note that the dependence on the number of the neglected years is not linear, most of the change in the mean difference is due to the drift between mid 2008 and mid 2009. The RMS for the drift displacement by 5 years reaches $6 \mathrm{~mm}$ in the radial, $15 \mathrm{~mm}$ in the tangential and $10 \mathrm{~mm}$ in the normal directions.

Figure 9 shows the RMS of the orbit fit for each solution. Even if the differences are only at the level of $10^{-4}-10^{-5} \mathrm{~mm} / \mathrm{s}$, the decrease in the RMS with lower number of the years with a neglected drift is

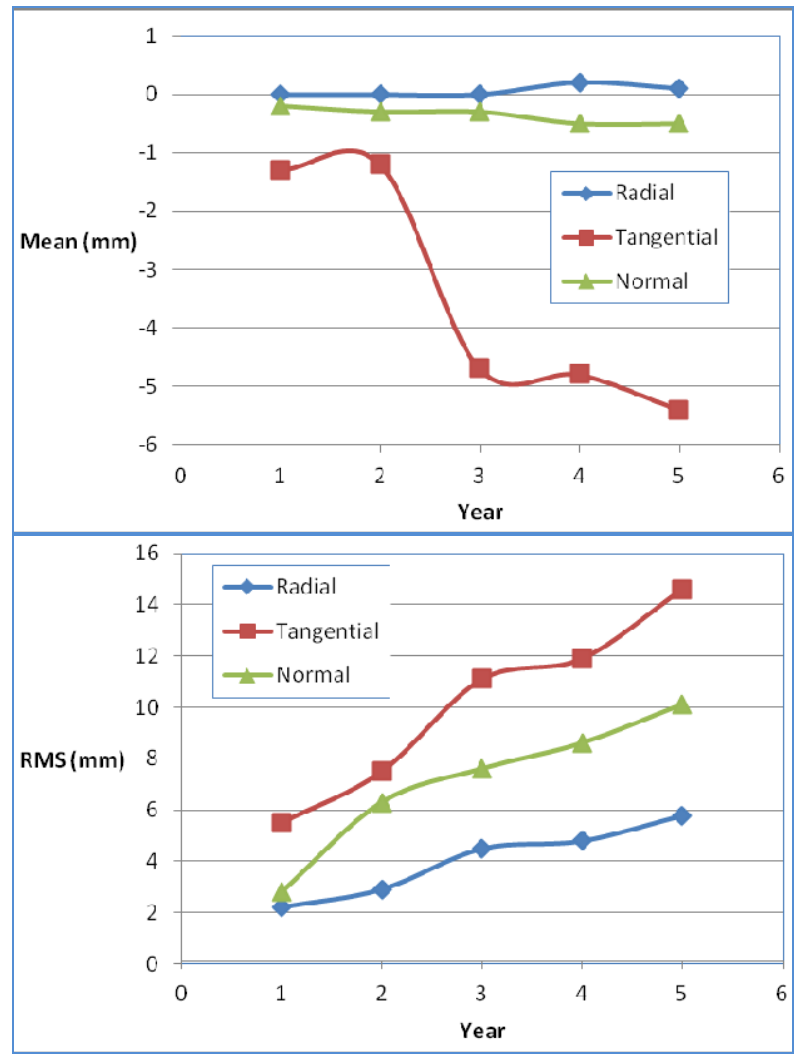

Fig. 8 The mean difference and RMS of the orbit differences estimated with shifting piecewise linear changes by 1-5 years compared to the nominal orbit (SPOT-5 DORIS data).

illustrated very clearly. This result shows, in agreement with Rudenko et al. (2014), that the inclusion of the annual drift has a positive impact on the solution accuracy.

The comparison of the solutions computed with the standard application of EIGEN-6S2 and with the application of EIGEN-6S2 referred to the time point 5 years before the POD epoch, was performed, also for the satellites Cryosat-2 and Jason-2. The results are summarized in Table 3. A lower impact on the Jason2 orbit is expected due to the higher satellite altitude. The impact on the Cryosat- 2 orbit is comparable to SPOT-5, while the RMS of the fit degradation due to the 5-year gravity field drift displacement is twice higher than for SPOT-5.

\subsubsection{ANNUAL AND SEMIANNUAL TERMS}

Current gravity field models provide the annual and semiannual terms, related to the periodical gravity field variations. For LEO satellites POD, the application of the periodical gravity terms is not one of the major conditions, radial orbit accuracy of 1$2 \mathrm{~cm}$ for DORIS satellites can be reached even without using these terms (e.g., Willis et al., 2010). However, IDS recommendations for the new ITRF DORIS data reprocessing request the use of the gravity field model including the periodical terms since 2002 at least (Willis et al., in press). As 
Table 3 Mean orbit difference, RMS of the orbit differences and RMS of the fit based on the gravity field model referenced to the time point 5 years before the observation epoch.

\begin{tabular}{lllllllllcr}
\hline & & \multicolumn{3}{c}{ Mean $(\mathrm{mm})$} & & \multicolumn{3}{c}{ RMS (mm) } & \multicolumn{3}{c}{ RMS of fit (mm/s) } \\
Satellite & Radial & Tangential & Normal & Radial & Tang. & Normal & Years & Years & diff. \\
\hline SPOT-5 & 0.1 & -5.4 & -0.5 & 5.8 & 14.6 & 10.1 & 0.4176 & 0.4182 & 0.0006 \\
Cryosat-2 & 0.2 & -3.6 & -0.4 & 7.1 & 17.2 & 11.8 & 0.4532 & 0.4545 & 0.0013 \\
Jason-2 & 0.1 & -2.8 & 0.1 & 2.2 & 7.6 & 6.4 & 0.4300 & 0.4300 & 0.0000 \\
\hline
\end{tabular}

Table 4 Mean and RMS of the orbit differences estimated without the application of the harmonic time-varying gravity terms, relative to the orbit estimated with the harmonic gravity terms included.

\begin{tabular}{l|ccc|ccc}
\hline & \multicolumn{3}{|c|}{ Mean (mm) } & \multicolumn{3}{c}{ RMS (mm) } \\
\hline Satellite & Radial & Along & Out & Radial & Along & Out \\
SPOT-5 & 0.0 & -0.1 & 0.0 & 1.1 & 3.6 & 3.2 \\
Cryosat-2 & 0.0 & -0.2 & 0.0 & 1.1 & 3.7 & 3.3 \\
HY-2A & 0.0 & 0.5 & 0.0 & 1.8 & 6.9 & 3.6 \\
\hline
\end{tabular}

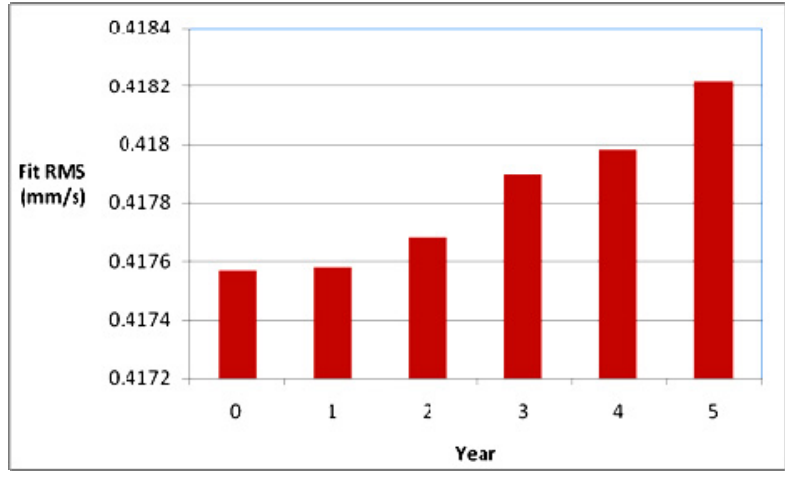

Fig. 9 RMS of the orbit fit based on the gravity field model referenced to the time point $0-5$ years before the observation epoch (SPOT-5 DORIS data).

mentioned in the Introduction, comparisons of the gravity field models application in the POD of DORIS satellites were performed, but a dedicated study discussing the impact of the harmonic terms has been missing.

For this experiment the satellite orbits were determined using the gravity model EIGEN-6S2, with and without periodical terms. The differences between the satellite orbits estimated using the gravity field with and without annual and semiannual terms are presented in Table 4. The tests were performed for satellites SPOT-5, Cryosat-2 and HY-2A. The mean differences in the radial and normal directions are under $0.1 \mathrm{~mm}$, while in the tangential direction the mean difference reaches $0.5 \mathrm{~mm}$. The RMS of the orbit differences after the mean removal is under $2 \mathrm{~mm}$ in the radial direction, under $7 \mathrm{~mm}$ in the tangential direction and under $4 \mathrm{~mm}$ in the cross-track direction. However, even if the annual mean differences are at the submilimeter level, the daily mean differences could be much larger, because the periodical time behavior is expected. For SPOT-5 the daily mean differences are displayed in Figure 10. In the radial and normal directions they stay well below $1 \mathrm{~mm}$. For the tangential direction, we clearly observe the signal with the amplitude of around $4 \mathrm{~mm}$. The behavior for Cryosat-2 and HY-2A is not presented, but it looks very similar.

The differences between the orbits estimated with and without the application of the periodical gravity model terms show the size of the changes in terms of the orbit ephemerides, but not the possible impact on the accuracy of their estimation. For this reason, other comparisons were performed, namely a comparison of the post-fit residual RMS and a comparison of the orbit arc overlap RMS (for midnight epochs). The results are summarized in Table 5. There is not any improvement in the post-fit residual RMS applying the periodical gravity terms, for the Cryosat-2 we observe even a degradation of $0.002 \mathrm{~mm} / \mathrm{s}$. For the arc overlaps RMS, we may observe a minor improvement, with the exception of the HY-2A tangential and Cryosat-2 radial components. In average, the overlap RMS decreased applying the periodical gravity terms by $0.6 \%$ in radial, $0.8 \%$ in tangential and $2.0 \%$ in normal component. In summary, the post-fit residuals test did not confirm POD accuracy improvement, arc overlap RMS generally showed a minor improvement.

\section{OCEAN TIDES}

This section is devoted to the application of the ocean tides (OT) model in the POD of DORIS satellites. We want to demonstrate the total effect on the estimated orbit ephemerides as well as to determine the required accuracy of the OT model application. Since the OT models are expressed by means of the geopotential harmonic coefficients and their variations, we are looking for a reasonable limit for the maximum degree of the OT harmonic coefficient series. We are not comparing various OT models, in our experiments we use only the FES2004 
Table 5 Post-fit residual RThe radial RMS of the estimated orbit relative to the SSALTO orbit (SPOT-5).

\begin{tabular}{llllll}
\hline Satellite & Harmonics & \multicolumn{3}{l}{ Post-fit residual RMS } & \multicolumn{3}{l}{ Arc overlaps RMS (mm) } \\
& & $\mathrm{mm} / \mathrm{s}$ & Radial & Along & Out \\
\hline SPOT-5 & excluded & 0.4062 & 18.3 & 134.3 & 47.9 \\
& included & 0.4062 & 18.2 & 133.7 & 47.3 \\
Cryosat-2 & excluded & 0.4578 & 30.7 & 125.9 & 70.1 \\
& included & 0.4598 & 30.8 & 122.9 & 67.1 \\
HY-2A & excluded & 0.4434 & 24.9 & 191.6 & 57.1 \\
& included & 0.4433 & 24.5 & 192.3 & 56.7 \\
\hline
\end{tabular}

ocean tide model. Other models are based on similar principles and results for them probably would not be much different. Our testing is based on 2 months of data, from 1 March to 31 April 2011. To demonstrate the total impact of the OT model application, the SPOT-5 orbit, estimated with and without an application of the OT model, was determined and compared with SSALTO (Segment-Sol multimissions d'ALTimétrie, Orbitographie et localisation précise) orbit (Cerri et al, 2010). The radial RMS of the determined orbit relative to the SSALTO orbit is displayed in Figure 11. For the orbit determined with the OT model applied, the RMS reaches $12 \pm 3 \mathrm{~mm}$. For the orbit determined without the OT model application, the RMS reaches more than twice the preceding value, $29 \pm 7 \mathrm{~mm}$. In this RMS time series we clearly observe the signal with a period around 14 days, correlating with the Moon phase. The RMS of the orbit fit decreased when applying the OT model from $0.4261 \mathrm{~mm} / \mathrm{s}$ to $0.4212 \mathrm{~mm} / \mathrm{s}$ and the decrement was observed for all the orbit arcs.

In the following experiment, we focus on two satellites with a different altitude, SPOT-5 and Jason2. Our interest is to understand how sensitive the orbit determination is to the ocean tides and how precisely the ocean tides should be modeled. The application of the ocean tides models is usually limited by a specified maximum degree of the corresponding geopotential coefficients, for which the change due to the ocean tides is calculated. Figure 12 displays the 3D size of the mean difference and RMS of the orbit differences for orbit estimated with various maximum degree limits of the OT spherical expansion (i.e. up to degree $0,4,8,12,20$ ) with respect to the reference orbit. As a reference, we used an orbit estimated with the application of the OT geopotential coefficients up to degree 50. It is clear from Figure 12 that neglecting the ocean tide effects leads to a mean difference at the millimeter level for both satellites $(4.5 \mathrm{~mm}$ for SPOT5 and $8.3 \mathrm{~mm}$ for Jason-2) and to an RMS at the centimeter/decimeter level $(4.6 \mathrm{~cm}$ for SPOT-5 and $9.3 \mathrm{~cm}$ for Jason-2). By adding the OT geopotential changes, the agreement with the reference orbit is strongly increasing. The mean difference reaches the values under $1 \mathrm{~mm}$ for degree limit 5-10 with Jason-2 and for degrees $10-15$ with SPOT-5. The mean under
$0.1 \mathrm{~mm}$ is reached with degrees $15-20$ for SPOT-5 and around degree 25 for Jason-2. The RMS reaches the values under $1 \mathrm{~mm}$ for degree limit around 20 with Jason-2 and around degree 25 with SPOT-5.

The previously described results are relevant for the standard dynamical model (Table 2). The reduceddynamical model using the empirical-stochastic approach could be less sensitive to the OT mismodeling, due to a higher number of estimated parameters possibly absorbing part of the modeling errors. Figure 13 is similar to Figure 12 and shows the size of the mean difference and the RMS of the orbit differences with a limited OT application relative to the reference orbit. Two plots are displayed, for an orbit approximated by standard dynamical model and for an orbit approximated by a reduced dynamical model (both for SPOT-5, see Table 2). Obviously, the reduced-dynamical orbit is less sensitive to mismodeling errors due to the total exclusion of the OT modeling. The size of the mean difference is $30 \%$ less and the RMS being about $40 \%$ less for the orbit approximated by the reduced-dynamic model. When applying the OT model up to degree 4 in the geopotential expansion, the mean orbit difference and the RMS values are by more than $70 \%$ less. When increasing the degree limit of the OT spherical expansion up to 20 , both orbit modeling approaches reach a similar size of the mean orbit difference and RMS.

\section{SUMMARY AND CONCLUSIONS}

The application of the gravity field in POD modeling is considered here in several ways. For the static gravity field and for the ocean tides, we were looking for a reasonable minimum limit on the spherical expansion application. For the time varying gravity, we studied the impact on the satellite orbit estimates and also the impact on the accuracy of the orbit determination, represented by the RMS of the fit and by the arc overlap RMS. The results of these experiments may help the DORIS analysts to optimize the applied strategy of gravity field modeling and to understand the size of the error introduced to their solutions when neglecting some of the gravity effects. For the time period outside the defining interval of the time varying gravity field model, the neglecting or 


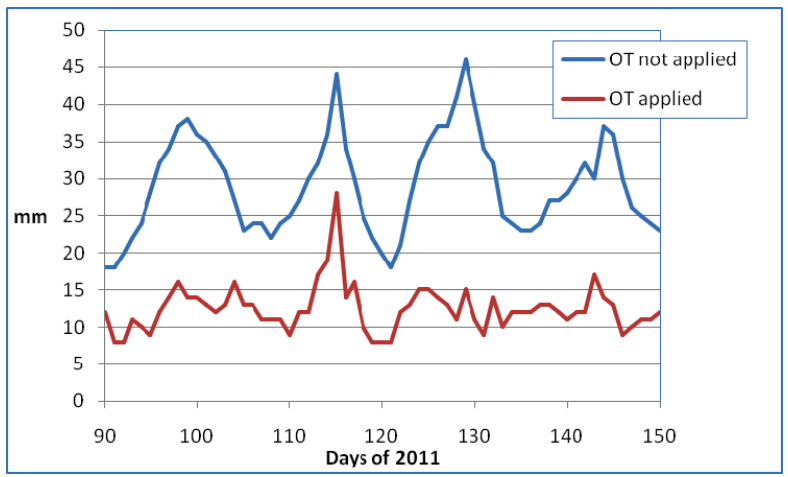

Fig. 11 The radial RMS of the estimated orbit relative to the SSALTO orbit (SPOT-5).
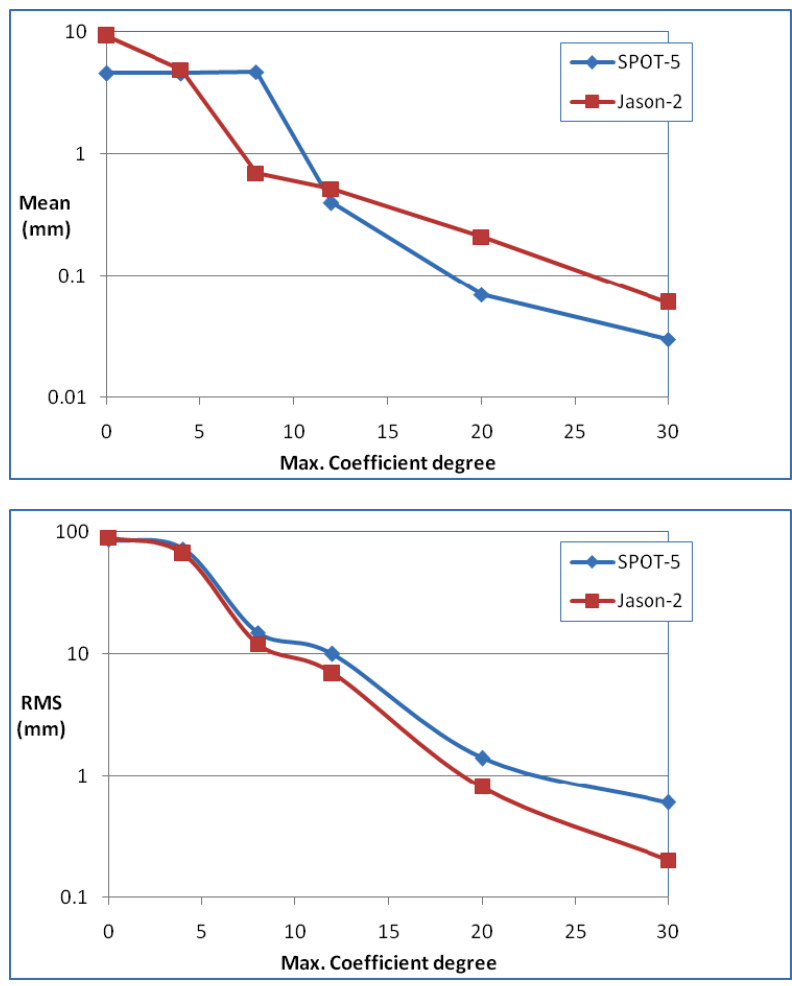

Fig. 12 The mean and RMS of the orbit differences for the orbit estimated with various maximum degree limits (up to degree $0,4,8,12,20$ ) with respect to the reference orbit.

inaccurate modeling of the time varying gravity cannot be avoided.

Based on the dynamic orbit parametrization from Table 2 ("standard dynamic") in the POD, we tried to find a minimum limit for the geopotential expansion to meet some reasonable accuracy requests. For testing, two satellites were chosen to represent two groups of satellites at different altitudes, SPOT-5 for satellites at altitudes of 700-1000 km and Jason-2 for satellites at an altitude of about $1300 \mathrm{~km}$. To find the limit, we compared the orbits estimated by applying the gravity field model with a changing degree limit. As reasonable values we considered the orbit
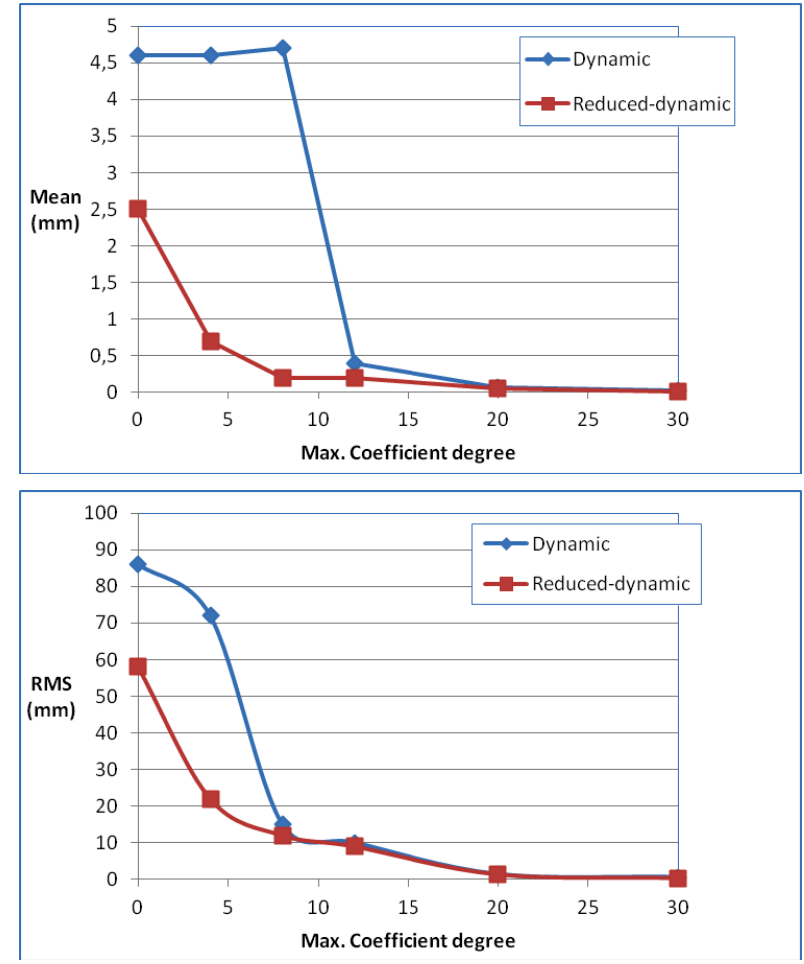

Fig. 13 The mean and RMS of the orbit differences for the SPOT-5 orbit estimated with various maximum degree limits (up to degree 0, 4,8 , $12,20)$ with respect to the reference orbit. Dynamic and reduced dynamic orbit models.

difference RMS values of $1 \mathrm{~mm}$ in the radial and of $2 \mathrm{~mm}$ in the along-track and cross-track directions and the mean difference one order of magnitude lower. These accuracy requirements were reached with the gravity field truncation of 75 for SPOT-5 and of 50 for Jason-2 at 1 day orbital arcs. The application of these limits saves about $25 \%$ of the computing time relative to the limit of 150 and about $11 \%$ of the computing time relative to the limit of 120 . For the application of the ocean tide model, the criteria of the acceptable orbit difference were set to $0.1 \mathrm{~mm}$ for the mean and $1 \mathrm{~mm}$ for the RMS of the orbit differences. These requirements at 1 day orbital arcs were fulfilled with the truncation limit of 25 on the OT spherical expansion for SPOT-5 and with the limit of 20 for Jason-2. However, it was shown that the truncation limit depends also on the orbit parametrization. Moreover, the tests for longer than daily arcs were not performed.

In agreement with Rudenko et al. (2014), we found an improvement by applying the piecewise linear change in the time-varying gravity modeling instead of using only the static gravity field models, as was demonstrated by the reduction in the RMS of the orbit fit. We showed a clear correlation between the growing RMS of the orbit fit estimated with the static gravity field on one side and the increasing time span between the reference point and the day of the observation on the other side. The differences were 
calculated between the orbit estimated with a piecewise linear time-varying gravity modeling and the orbit estimated with the static gravity field with the reference point defined 5 years before the DORIS data epoch. For this experiment the data from satellites SPOT-5, Cryosat-2 and Jason-2 were used. The mean orbit differences reached the submilimeter values in the radial and normal directions and millimeter values in the tangential direction. A similar study was performed also for the harmonic terms of the time-varying gravity field model, but the POD improvement was confirmed only from orbit overlap RMS (minor reduction of 0.6-2.0\%), but not from the RMS of the orbit fit. When comparing the orbits estimated with and without the application of harmonic gravity terms (SPOT-5, Cryosat-2, Hy-2A), the daily mean orbit differences reach a millimeter level in the tangential direction and a submilimeter level in the radial and tangential directions.

\section{ACKNOWLEDGMENTS}

The authors acknowledge IDS for DORIS data and other support, ESA for Swarm mission data, ICGEM/GFZ for the geopotential models. The work was supported by grant projects GACR/DFG15-24730J (P. Štěpánek, V. Filler) and LG14026 (A. Bezděk).

\section{REFERENCES}

Austen, G., Grafarend, W. and Reubelt, T.: 2002, Analysis of the Earth's gravitational field from semi-continuous ephemeris of a low Earth orbiting GPS-Tracked Satellite of Type CHAMP, GRACE or GOCE. IAG Symposia 2002, 125, 309-315.

Balmino, G., Perosanz, F., Rummel, R., Sneeuw, N. and Sunkel, H.: 1999, Champ, GRACE and GOCE: mission concepts and simulations. Bollettino di Geofisica Teoretica ed Applicata, 40, No. 3-4, 309311.

Bezděk, A.: 2010, Calibration of accelerometers aboard GRACE satellites by comparison with POD-based non-gravitational accelerations. Journal of Geodynamics, 50, No. 5, 410-423. DOI: $10.1016 /$ j.jog.2010.05.001

Bezděk, A., Klokočník, J., Kostelecký, J., Floberghagen, R. and Gruber, C.: 2009, Simulation of free fall and resonances in the GOCE mission. Journal of Geodynamics, 48, No. 1, 47-53. DOI: 10.1016/j.jog.2009.01.007

Bezděk, A., Sebera, J., Klokočník, J. and Kostelecký, J.: 2014, Gravity field models from kinematic orbits of CHAMP, GRACE and GOCE satellites. Advances in Space Research, 53, 412-429.

DOI: 10.1016/j.asr.2013.11.031

Bezděk, A. and Vokrouhlický, D.: 2004, Semianalytic theory of motion for close-Earth spherical satellites including drag and gravitational perturbations. Planet. Space Science, 52, No. 14, 1233-1249. DOI: $10.1016 /$ j.pss.2004.08.004

Cazenave, A. and Chen, J.: 2010, Time-variable gravity from space and present-day mass redistribution in the Earth system. Earth and Planetary Science Letters, 298, 263-274.

DOI: 10.1016/j.eps1.2010.07.035
Cerri, L., Berthias, J.P., Bertiger, W.I., Haines, B.J., Lemoine, F.G., Mercier, F., Ries, J.C., Willis, P. and Ziebart, M.: 2010, Precision Orbit Determination Standards for the Jason Series of Altimeter Mission In: Special Issue on OSTM/Jason-2 Calibration/Validation. Marine Geodesy, 33(S1), 379418. DOI: $10.1080 / 01490419.2010 .488966$

Chambers, D. P. and Schröter, J.: 2011, Measuring ocean mass variability from satellite gravimetry. Journal of Geodynamics, 52, No. 5, 333-343. DOI: $10.1016 /$ j.jog.2011.04.004

Chen, J. L., Wilson, C. R. and Tapley, B. D.: 2005. Interannual variability of low-degree gravitational change, 1980-2002. Journal of Geodesy, 78, No. 9, 535-543. DOI: 10.1007/s00190-004-0417-y

Cheng, M., Ries, J. C. and Tapey, B. D.: 2011, Variations of the Earth's figure axis from satellite laser ranging and GRACE. Journal of Geophysical Research, 116, B01409. DOI: 10.1029/2010JB000850

Couhert, A., Cerri, L., Legeais, J.F., Ablain, M., Zelensky, N.P., Haines, B.J., Lemoine, F.G., Bertiger, W.I., Desai, S.D. and Otten, M.: 2015, Towards the $1 \mathrm{~mm} / \mathrm{y}$ stability of the radial orbit error at regional scales. Advances in Space Research, 55, No. 1, 2-23. DOI: 10.1016/j.asr.2014.06.041

Dach, R., Hugentobler, U., Fridez, P. and Meindl, M.: 2007, Bernese GPS software version 5.0. Astronomical Institute, University of Bern, 640.

Dagras, C. H., Duran, M., Zarrouati, O. and Fratter, C.: 1995, The SPOT-5 mission. Acta Astronautica, 35, Nos. 9-11, 651-660. DOI: 10.1016/0094-5765(95)00016-S

Floberghagen, R., Fehringer, M., Lamarre, D., Muzi, D., Frommknecht, B., Steiger, C., Pineiro, J. and da Costa, A.: 2011, Mission design, operation and exploitation of the gravity field and steady-state ocean circulation explorer mission. Journal of Geodesy, 85, No. 11, 749-758. DOI: 10.1007/s00190-011-0498-3

Fukushima, T.: 2013, Recursive computation of oblate spheroidal harmonics of the second kind and their first-, second-, and third-order derivatives. Journal of Geodesy, 87, No. 4, 303-309.

DOI: $10.1007 / \mathrm{s} 00190-012-0599-7$

Gobinddass, M.L., Willis, P., Menvielle, M. and Diament, M.: 2010, Refining DORIS atmospheric drag estimation in preparation of ITRF2008. In Special Issue: Precise Orbit Determination and Applications to the Earth Sciences, P. Willis (Ed.). Advances in Space Research, 46, No. 12, 1566-1577.

DOI: $10.1016 /$ j.asr.2010.04.004

Kostelecký, J., Bezděk, A. and Klokočník, J.: 2013, Global and regional seasonal variations of the geoid detected by GRACE. Acta Geodyn. Geomater., 10, No. 3, 285291. DOI: $10.13168 /$ AGG.2013.0028

Lambin, J., Morrow, R., Fu Lee-Lueng, et al.: 2010, The OSTM/Jason-2 Mission. Marine Geodesy, 33, No. 1, 4-25. DOI: 10.1080/01490419.2010.491030

Le Bail, K., Lemoine, F.G. and Chinn, D.S.: 2010, GSFC contribution to ITRF2008. In DORIS Special Issue: Scientific Applications in Geodesy and Geodynamics, P. Willis (Ed.). Adv. Sp. Res., 45, No. 12, 1481-1499. DOI:10.1016/j.asr.2010.01.030

Lemoine, F.G., Bertiger, W., Beall, J., Zelensky, N.P. and Le Bail, K.: 2014, The development of the GSC DORIS contributions to ITRF2013. IDS DORIS Workshop presentation, Konstanz, http://idsdoris.org/images/documents/report/ids_workshop_201 4/IDS14_s1_Lemoine_GSCcontributionITRF2013.pdf 
Lemoine, F. G. and GSC Analysis Team: 2013, GSC analysis center report. IDS AWG meeting presentation, Toulouse,

http://ids-doris.org/images/documents/report/AWG20 1304/IDSAWG1304-LemoineGSCreport.pdf

Letellier, T., Lyard, F. and Lefevre, F.: 2004, The new global tidal solution: FES2004. Proceedings of the Ocean Surface Topography Science Team Meeting, St. Petersburg, Florida, 4-6 November.

Melachroinos, S.A., Lemoine, F.G., Chinn, D.S, Zelensky N. P., Nicholas, J. B. and Beckley, B. D.: 2014, The effect of seasonal and long-period geopotential variations on the GPS orbits. GPS Solutions, 18, No. 4, 497-507. DOI: $10.1007 / \mathrm{s} 1029-013-0346-4$

Montenbruck, O. and Gill, E.: 2000, Satellite Orbits: models, methods and applications. Berlin, Springer, $369 \mathrm{pp}$.

Nerem, R.S., Tapley, B.D. and Shum, C.K: 1990, Determination of the ocean circulation using Geosat altimetry. Journal of Geophysical Research: Oceans, 95(C3), 3163-3179.

DOI: $10.1029 / \mathrm{JC} 095 \mathrm{iC} 03 \mathrm{p} 03163$

Olsen, N., Hulot, G., Lesur V. et al.: 2015, The swarm initial field model for the 2014 geomagnetic field. Geophysical Research Letters 42, 1092-1098. DOI: $10.1002 / 2014$ GL062659

Otten, M., Flohrer, C., Springer, T. and Dow, J.: 2010, DORIS processing at the European Space Observations Centre. In DORIS Special Issue: Precise Orbit Determination and Applications to the Earth Sciences, P. Willis (Ed.). Advances in Space Research, 6, No. 12, 1606-1613. DOI: $10.1016 / j$.asr.2010.04.024

Pail, R. et al.: 2010, Combined satellite gravity field model GOCO01S derived from GOCE and GRACE. Geophys. Res. Lett., 37, L20314. DOI :10.1029/2010GL044906.

Pavlis, N.K., Holmes, S.A., Kenyon, S.C. and Factor, J.K.: 2012, The development and evaluation of the Earth gravitational model 2008 (EGM2008). Journal of Geophysical Research (Solid Earth), 117, B04406. DOI: 10.1029/2011JB008916

Petit, G. and Luzum, B.: 2010, IERS conventions. IERS Tech. Note, $36,1$.

Rapp, R. H. and Pavlis, N.K.: 1990, The development and analysis of geopotential coefficient models to spherical harmonic degree 360. Journal of Geophysical Research, 95(B13), 21885-21911. DOI: $10.1029 / J B 095 i B 13$ p21885

Ray, R. D., Rowlands, D. D. and Egbert, G. D.: 2003, Tidal models in a new era of satellite gravimetry. Space Science Reviews, 108, Nos. 1-2, 271-282. DOI: 10.1023/A:1026223308107

Reigber, C., Schwintzer, P., Neumayer, K.H. et al.: 2003, The CHAMP-only Earth gravity field model EIGEN2. Advances in Space Research 31:1883-1888. DOI: $10.1016 / \mathrm{S} 0273-1177(03) 00162-5$

Rudenko, S., Dettmering, D., Esselborn, S., Schöne, T., Förste, C., Lemoine, J.M., Ablain, M., Alexandre, D. and Neumayer, K.H.: 2014, Influence of time variable geopotential models on precise orbits of altimetry satellites, global and regional mean sea level trend, Advances in Space Research, 54(1), 92-118, DOI: $10.1016 /$ j.asr.2014.03.010
Sebera J., Wagner, C.A., Bezděk, A. and Klokočník, J.: 2013, Short guide to direct gravitational field modelling with Hotine's equations. Journal of Geodesy, 87, 223-238. DOI: $10.1007 / \mathrm{s} 00190-012-0591-2$

Shum, C.K., and 16 colleagues: 1997, Accuracy assessment of recent ocean tide models. Journal of Geophysical Research, 102, C11, 25173-25194. DOI: $10.1029 / 97 J C 00445$

Štěpánek, P., Douša, J., Filler, V. and Hugentobler, U.: 2010a, DORIS data analysis at Geodetic Observatory Pecny using single-satellite and multi-satellite geodetic solutions. In DORIS Special Issue: Precise Orbit Determination and Applications to the Earth Sciences, P. Willis (Ed.). Advances in Space Research, 46, No. 12, 1578-1592. DOI: 10.1016/j.asr.2010.04.015

Štěpánek, P., Filler, V., Hugentobler, U. and Douša, J.: 2010b, DORIS at GOP, From pilot testing campaign to fully operational analysis center. Acta Geodyn. Geomater., 7, No. 1, 49-60.

Štěpánek, P., Rodriguez-Solano, C.J., Hugentobler, U. and Filler, V.: 2014, Impact of orbit modeling on DORIS station position and Earth rotation estimates. Advances in Space Research, 53, No. 7, 1058-1070. DOI: 10.1016/j.asr.2014.01.007

Tapley, B.D., Bettadpur, S., Watkins, M. and Reigber, C.: 2004, The gravity recovery and climate experiment: mission overview and early results. Geophysical Research Letters 31, L09607. DOI: $10.1029 / 2004$ GL019920

Touboul, P., Willemont, E., Fouloun, B. and Josselin, V.: 1999, Accelerometers for CHAMP, GRACE and GOCE space missions: synergy and evolution. Bollettino di Geofisica Teoretica ed Applicata, 40, No. 3-4, 321-327.

Vallado, D.A.: 2006, Perturbed motion. In: Gurfil, P. (ed). Modern astrodynamics, Elsevier, 1-22.

Weigelt, M., Dam, T., Jäggi, A., Prange, L., Tourian, M. J., Keller, W. and Sneeuw, N.: 2013, Time-variable gravity signal in Greenland revealed by high-low satellite-to-satellite tracking. Journal of Geophysical Research (Solid Earth), 118, No. 7, 3848-3859. DOI: 10.1002/jgrb.50283

Willis, P., Fagard, H., Ferrage, P. et al.: 2010, The International DORIS Service, Toward maturity. In DORIS: Scientific Applications in Geodesy and Geodynamics, P. Willis (Ed.). Advances in Space Research, 5, No. 12, 1408-1420.

DOI: 10.1016/j.asr.2009.11.018

Willis, P. and Kuzin, S.: 2014, DORIS/IGN and INASAN Analysis Center Report. IDS AWG meeting presentation, Paris, idsdoris.org/images/documents/report/AWG201403/IDS AWG1403-Willis-IGN\&INASANStatusReport.pdf

Willis, P., Lemoine, F.G., Moreaux, G. et al.: 2015, The International DORIS Service (IDS), recent developments in preparation for ITRF2013. IAG Symposia Series, 143. 


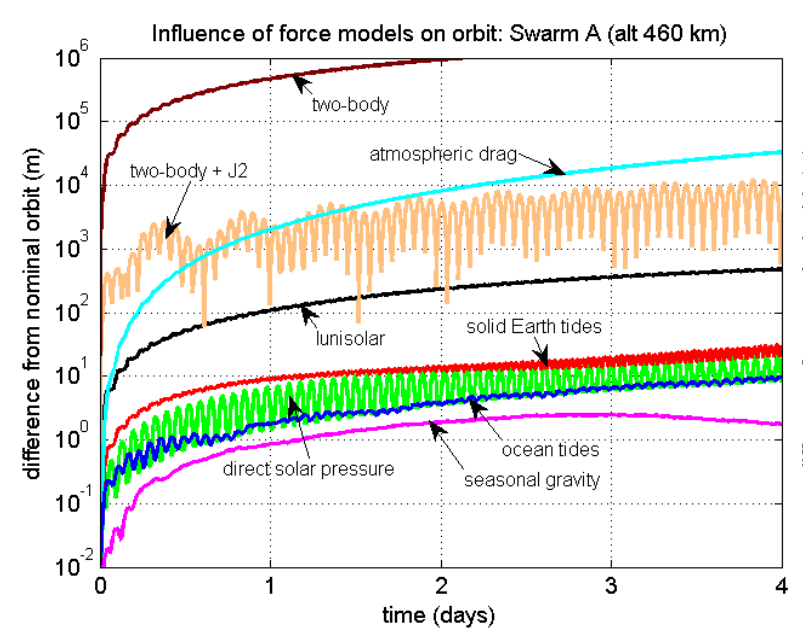

Fig. 1 Comparison of individual perturbing forces acting on the satellite Swarm A, the difference in the position with respect to a nominal orbit is shown as a function of time.

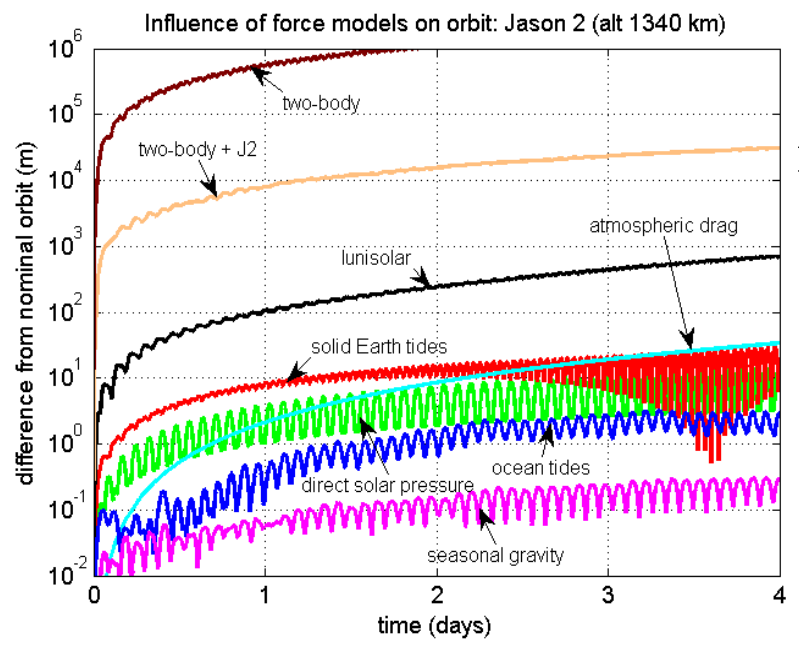

Fig. 3 Comparison of individual perturbing forces for the satellite Jason-2.

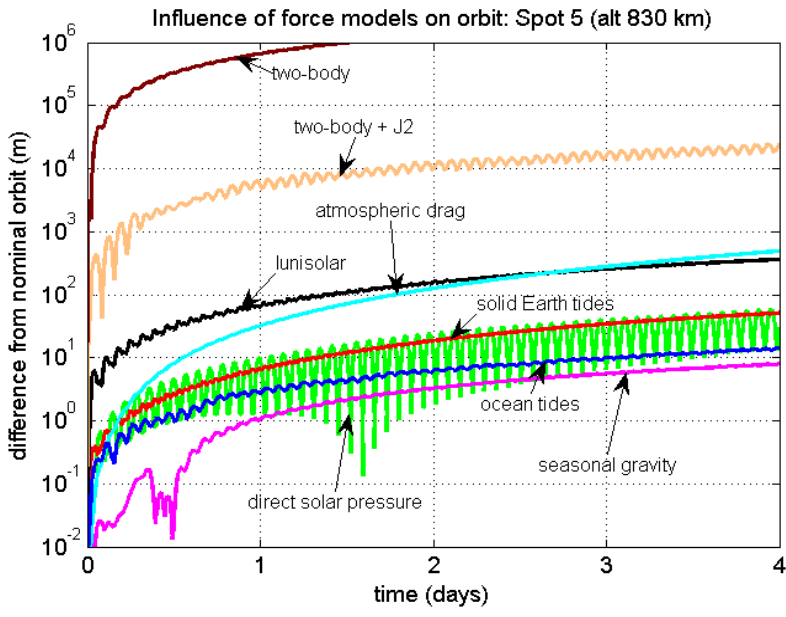

Fig. 2 Comparison of individual perturbing forces for the satellite SPOT-5.

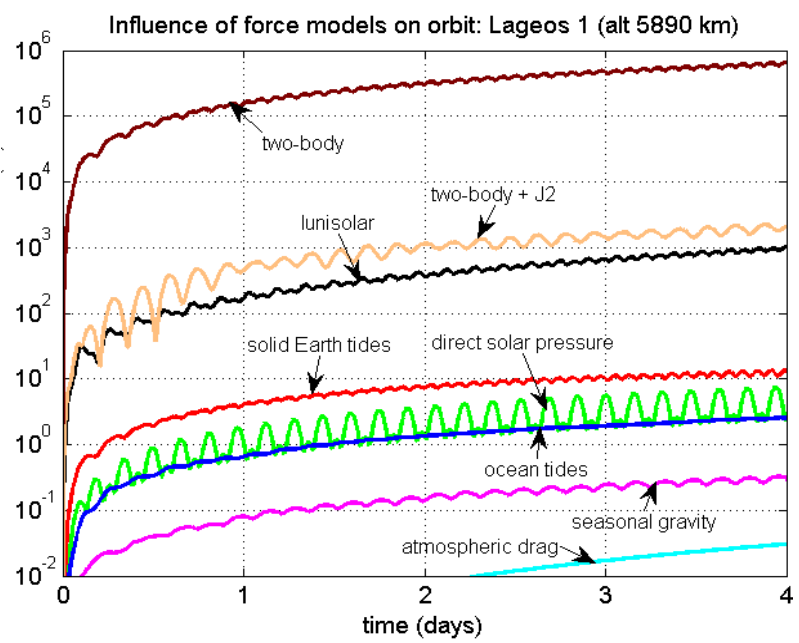

Fig. 4 Comparison of individual perturbing forces for the satellite Lageos-1.

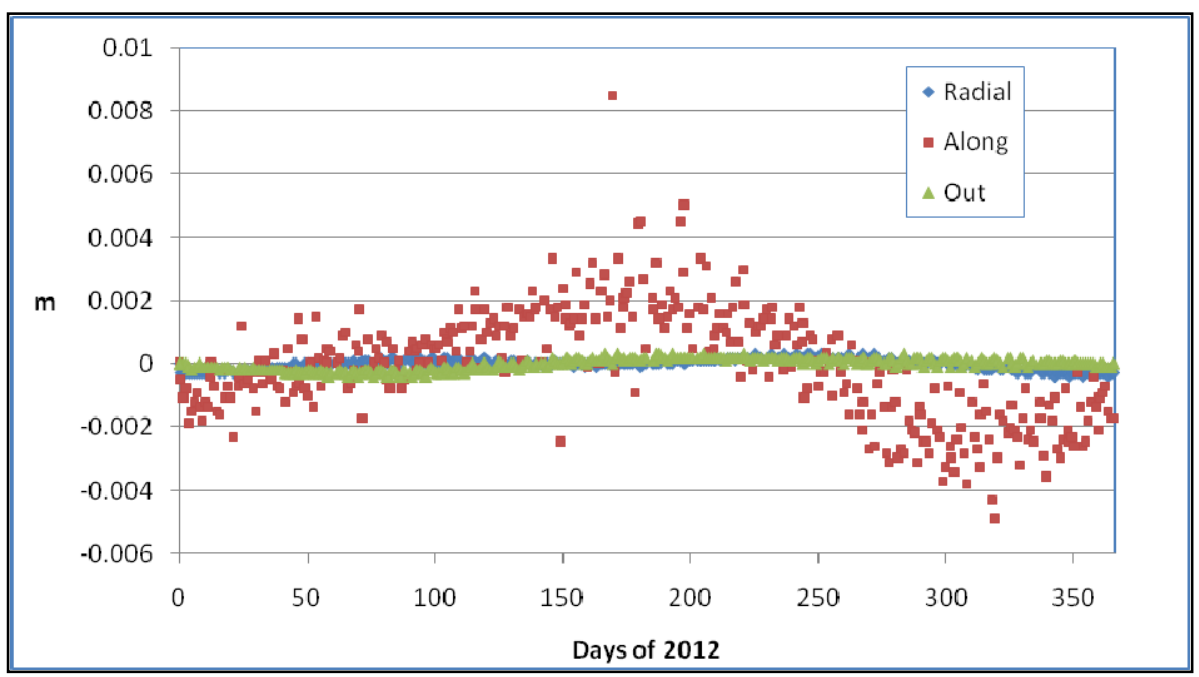

Fig. 10 Mean difference of the SPOT-5 orbit estimated without the application of the harmonic time-varying gravity terms, relative to the orbit estimated with the harmonic gravity terms included. 